\title{
Mesoscopic structures and the Laplacian spectra of random geometric graphs
}

\author{
Amy Nyberg, ${ }^{1,2}$ Thilo Gross ${ }^{3}$ and Kevin E. Bassler ${ }^{1,2,4}$ \\ ${ }^{1}$ Department of Physics, University of Houston, Houston, Texas 77204-5005, USA \\ ${ }^{2}$ Texas Center for Superconductivity, University of Houston, Houston, Texas 77204-5002, USA \\ ${ }^{3}$ Department of Engineering Mathematics, University of Bristol, \\ Merchant Venturers Building, Woodland Road, Bristol BS81UB, UK \\ ${ }^{4}$ Max Planck Institute for the Physics of Complex Systems, \\ Nöthnitzer Straße 38, Dresden D-01187, Germany
}

(Dated: August 17, 2021)

\begin{abstract}
We investigate the Laplacian spectra of random geometric graphs (RGGs). The spectra are found to consist of both a discrete and a continuous part. The discrete part is a collection of Dirac delta peaks at integer values roughly centered around the mean degree. The peaks are mainly due to the existence of mesoscopic structures that occur far more abundantly in RGGs than in non-spatial networks. The probability of certain mesoscopic structures is analytically calculated for one-dimensional RGGs and they are shown to produce integer-valued eigenvalues that comprise a significant fraction of the spectrum, even in the large network limit. A phenomenon reminiscent of Bose-Einstein condensation in the appearance of zero eigenvalues is also found.
\end{abstract}

Over the past two decades there has been considerable progress in the development of parameters and measurements to characterize complex networks. This has resulted in a rich description of both the microscopic and macroscopic properties of networks [1-3]. However, until recently the intermediate, or mesoscale, level has not received the same degree of attention [4, 5]. The mesoscale level, though, is particularly interesting because it is there that one can begin to understand how a network's modular structure affects its dynamics. From studies of the relationship between graph spectra and the structure and dynamics of networks [6 9] it is known that certain mesoscale structures, namely symmetric (or quasi-symmetric) motifs reveal themselves in the spectrum [10]. Symmetric motifs are of particular interest [1] because their spectral properties imply that the presence of a single such motif in a given network can have distinct, well-defined consequences for systemlevel processes such as diffusion, synchronization, or more complex dynamics [12 15]. While symmetric structures are relatively rare in random non-spatial graphs, here we show that they occur abundantly in random geometric graphs (RGGs). RGGs result from randomly placing $N$ vertices in space and connecting those that are close and differ from other random graph models because of the metric that defines a distance between vertices [16, 17]. They are commonly used for modeling spatially embedded systems [18, 19] such as wireless networks 20], transportation and power grids 21], neural networks [22, 23], and certain biological processes [24, 25]. Here, we show that the ensemble-averaged spectra of the graph Laplacian matrices of RGGs indicate that mesoscopic symmetric structures occur abundantly in these graphs.

An example of a $2 \mathrm{~d}$ RGG embedded on a square is shown in Fig. 1. Though to make progress analytically, we focus mostly on the Laplacian spectrum of 1d RGGs embedded on the unit circle, that is, in the domain $[0,1]$

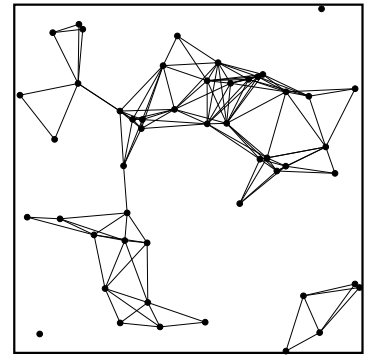

FIG. 1: A RGG embedded in 2d on a square.

with periodic boundary conditions. Within this domain, the vertices are distributed randomly with uniform probability and two vertices are connected when the Euclidian distance between them is smaller than a threshold distance $r$. The discretized Laplace operator on the graph is given by the Laplacian matrix $\mathcal{L}$, which has elements $\mathcal{L}_{i j}=k_{i} \delta_{i j}-a_{i j}$, where $k_{i}$ is the degree of vertex $i$ and $a_{i j}$ is an element of the adjacency matrix, i.e., $a_{i j}=1$ if vertices $i$ and $j$ are connected and 0 otherwise. For this one-dimensional case, we analytically calculate the proportion of eigenvalues due to symmetry in the extensive and intensive scaling limits of large graphs. Additionally, investigating the occurrence of the particular eigenvalue $\lambda=0$, we identify a phenomenon that is reminiscent of Bose-Einstein condensation.

As can be seen in the example shown in Fig. 2, the spectra consist of discrete, Dirac delta peaks at integer eigenvalues and a broad distribution of eigenvalues between the integers. The part of the spectra between the integers becomes continuous in the large network limit. We refer to the Dirac delta peaks as the discrete part of the spectrum and the remainder as the continuous part. Here, we focus on the discrete part. As this figure suggests, and as we will analytically prove for 1d RGGs, the discrete eigenvalues comprise a finite fraction of the spec- 


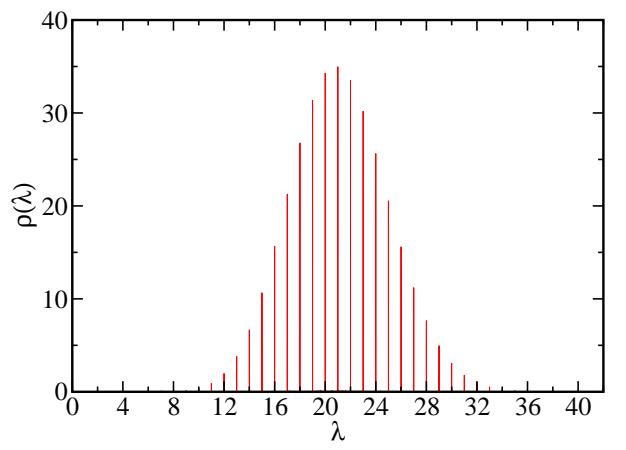

(a)

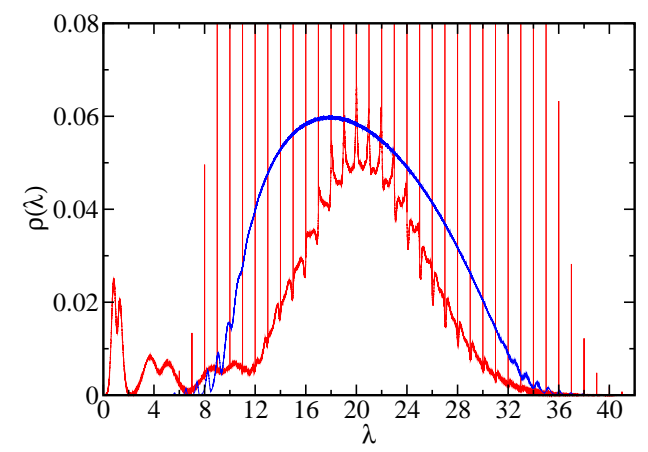

(b)

FIG. 2: (Color online) The ensemble-averaged Laplacian spectrum for 1d RGGs on the unit circle (red), with $N=100$ and $r=0.1$. (a) Top figure shows the discrete, Dirac delta peaks that exist at integer eigenvalues. The envelope of these peaks is centered about $\langle k\rangle+1$ where $\langle k\rangle=20$ is the mean degree of the network. (b) The bottom figure is a zoom-in of the top figure. Between the discrete peaks, the spectrum has a continuous part. The ensemble-averaged Laplacian spectum for Erdős-Rényi graphs with the same $N$ and $\langle k\rangle$ is shown for comparison (blue). These results are obtained from numerical diagonalization of $10^{6}$ realizations. A bin size of $\Delta \lambda=0.001$ was used to construct the histogram.

tra. Note that the height of the Dirac delta peaks, but not the continuous part of the spectra, depend on the bin size used. The relative height of the peaks and, thus, the shape of their envelope is, however, independent of bin size. By contrast, similar peaks are not visible in the ensemble-averaged Laplacian spectra of non-spatial random graph models such as the Erdős-Rényi graphs, as the example shown in Fig. 2 indicates.

The network structure that causes a given eigenvalue can be identified by considering the corresponding eigenvector. In an RGG, the eigenvectors of many of the integer eigenvalues are exactly localized on symmetric motifs such that the eigenvector's only nonzero components correspond to vertices of the motif. Such motifs, where the adjacency of the vertices is invariant under a permutation of indices, are called graph orbits 10]. Orbits leading to integer eigenvalues are significantly more frequent in spatial networks, such as RGGS, because of the geographical

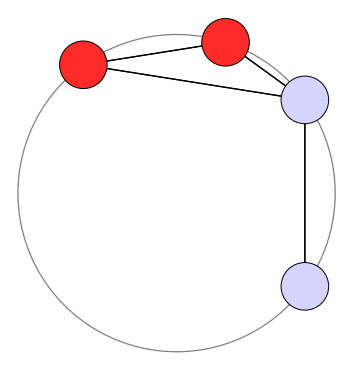

(a)

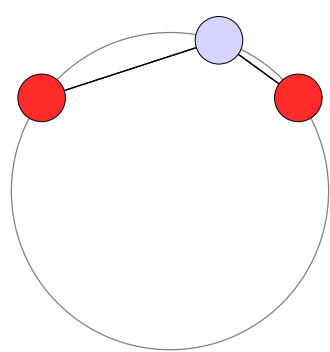

(b)
FIG. 3: (Color online) A Type-I orbit (a) and a Type-II orbit (b) in 1d RGGs on the unit circle. Vertices in an orbit are colored red. Vertices in Type-I orbits are connected to each other, but vertices in Type-II orbits are not.

proximity of neighbors. The simplest type of orbit consists of a single set of vertices that can all be permuted. If the vertices in such a simple orbit are all connected to each other, so that they form a clique, we call it a Type-I orbit. If they are not connected to each other then we call it a Type-II orbit. Simple examples of Type I and Type II orbits are shown in Fig. 3. More complicated types of orbits are also possible, but are rare in RGGs.

Type-I and Type-II orbits account for the vast majority of the integer eigenvalues found in the Laplacian spectra of RGGs. Since eigenvectors localize on the orbits, the corresponding eigenvalue is independent of the embedding network. Type-I orbits produce an eigenvalue equal to one more than the degree of the vertices in the orbit, while the eigenvalues resulting from Type-II orbits are equal to their degree 26]. To see this, let $i$ and $j$ be two vertices in a simple orbit, and $\mathbf{x}$ be a vector with components $x_{i}=1, x_{j}=-1$, and all others zero. If $k$ is the degree of the vertices in the orbit, then $\mathcal{L} \mathbf{x}=(k+1) \mathbf{x}$ for Type-I and $\mathcal{L} \mathbf{x}=k \mathbf{x}$ for Type-II. An orbit of size $n$ has $n-1$ of these independent and orthogonal eigenvectors.

To compute the expected number of simple orbits in 1d RGGs we begin by defining the following terms. The geographical neighborhood $\mathcal{N}(i)$ of a vertex $i$ is the region within a distance $r$ of the vertex. For a pair of vertices, their shared neighborhood $\mathcal{N}_{\mathrm{s}}(i, j)=\mathcal{N}(i) \cap \mathcal{N}(j)$ is the common region that is in the geographical neighborhood of both vertices and their excluded neighborhood $\mathcal{N}_{\text {ex }}(i, j)=(\mathcal{N}(i) \cup \mathcal{N}(j)) \backslash(\mathcal{N}(i) \cap \mathcal{N}(j))$ is the region that is in the geographical neighborhood of one vertex, but not the other.

The average multiplicity of eigenvalues due to TypeI orbits can be calculated by considering each of the $N(N-1) / 2$ pairs of vertices and calculating the likelihood that they are nearest neighbors with exactly $k-2$ vertices in their shared neighborhood and no vertices in their excluded neighborhood. Such a motif produces an integer eigenvalue equal to $k$. Requiring that the pair is a nearest neighbor pair ensures that the correct mul- 
tiplicity of the eigenvalues is obtained because in a $1 d$ RGG orbits that are chains of size $n$ have $n-1$ nearest neighbor pairs. Then, for $r \leq \frac{1}{3}$, the expected number of eigenvalues $\lambda=k$ is

$$
\begin{aligned}
E_{1}(k)=\frac{N ! r}{}(2 r)^{k-2}(1-2 r)^{N-k} \\
(k-1) !(N-k) ! \\
\quad \times{ }_{2} F_{1}\left[1, k-N, k, \frac{r}{1-2 r}\right],
\end{aligned}
$$

where ${ }_{2} F_{1}$ is the ordinary hypergeometric function. Because vertices are distributed randomly with uniform probability in RGGs, the probability that a particular number of them are in a region of a given size is given by the binomial distribution. Then, noting that Type-I orbits can produce eigenvalues ranging from 2 to $N$, the total number of integer eigenvalues due to Type-I orbits is

$$
T_{1}=\sum_{k=2}^{N} E_{1}(k)=\frac{N}{3}\left[1-(1-3 r)^{N-1}\right]
$$

In 1d RGGs, Type-II orbits and their shared neighbors always consist of an entire component of the graph. The average multiplicity of eigenvalues due to Type-II orbits can be calculated similarly to those due to Type-I orbits, except that now one must calculate the probability that a pair of vertices not connected to each other share exactly the same $k$ neighbors. For $r \leq \frac{1}{4}$, the expected number of eigenvalues $\lambda=k$ is

$$
\begin{aligned}
E_{2}(k)=\frac{N ! r^{k+1}(1-3 r)^{N-2-k}}{(k+1) !(N-2-k) !} \\
\quad \times{ }_{2} F_{1}\left[1, k+2-N, k+2, \frac{r}{1-3 r}\right] .
\end{aligned}
$$

Type-II orbits can produce eigenvalues ranging from 0 to $N-2$, thus the total number of integer eigenvalues due to Type-II orbits is

$$
T_{2}=\sum_{k=0}^{N-2} E_{2}(k)=\frac{N}{2}\left[(1-2 r)^{N-1}-(1-4 r)^{N-1}\right](4)
$$

Finally, in addition to the $\lambda=0$ eigenvalues due to Type-II orbits in which two vertices are between $r$ and $2 r$ apart and their shared neighborhood is empty, there is an extra contribution of eigenvalue $\lambda=0$ in the spectra that occurs when pairs of vertices more than $2 r$ apart have no edges at all. Such pairs are too far apart to have a shared neighborhood. The expected number of these extra eigenvalues is

$$
E_{*}(0)=(N-1)(1-4 r)^{N-1} .
$$

The contribution to the spectrum is analogous to the accumulation of particles in the lowest energy level in Bose-Einstein condensation. In the analogy, $r$ plays the

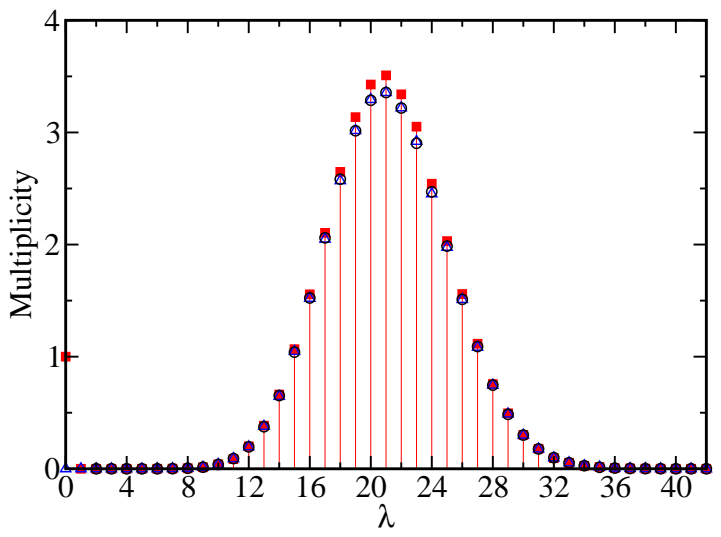

FIG. 4: (Color online) Ensemble-averaged integer eigenvalue multiplicity for $1 \mathrm{~d}$ RGGs with $N=100$ and $r=0.1$. The red points are the results of numerical diagonalization of $10^{6}$ random ensemble realizations and the black points are the number due to Type-I orbits according to Eq. 1

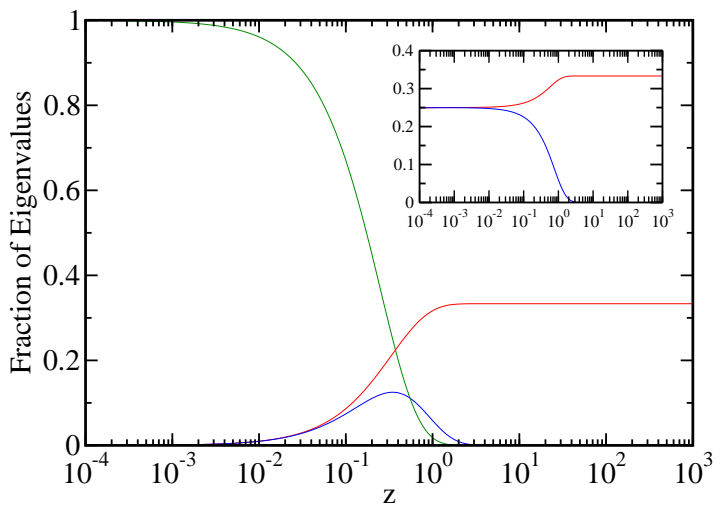

FIG. 5: (Color online) Fraction of eigenvalues due to Type-I orbits (red), Type-II orbits (blue), and in the $\lambda=0$ condensate (green) in the extensive large network limit as a function of $z=N r$. Inset shows the fraction of eigenvalues not in the $\lambda=0$ condensate that are due to Type-I (red) and Type-II (blue) orbits.

role of temperature and the eigenvalues are the energy levels. Note that $\lim _{r \rightarrow 0} E_{*}(0)=N-1$.

Figure 4 shows a comparison of the average multiplicity of integer eigenvalues for an ensemble of 1d RGGs found using numerical diagonalization with the number due to Type-I orbits predicted analytically using Eq. 1. In the case considered, $N=100$ and $r=0.1$, the total number of eigenvalues due to Type-I orbits is $\sim 33.3$, which is $\sim 1 / 3$ of all eigenvalues. Note that for this case the Type-I orbits account for the vast majority of integer eigenvalues, as there are only $\sim 10^{-10}$ expected to be caused by Type-II orbits and only $\sim 10^{-22}$ expected to be in the condensate of extra $\lambda=0$ eigenvalues. The discrepancy between the theoretical and numerical curves in Fig. 4 arises due to the presence of orbits with more complex symmetries and other mechanisms [27].

The fraction of the spectra due to simple orbits in 
large network limits can be calculated using Eqs. 2 and 4. In the intensive limit of large $N$ and fixed $r$, for all $r, \lim _{N \rightarrow \infty} \frac{1}{N} T_{2}=0$ because there are no Type-II orbits, but there are many Type-I orbits and the fraction of corresponding eigenvalues is

$$
\lim _{N \rightarrow \infty} \frac{1}{N} T_{1}=\frac{1}{3}
$$

Thus, the discrete part of the spectrum comprises a substantial finite fraction of the total number of eigenvalues, even in the large network limit.

Perhaps a more important thermodynamic limit though is the extensive limit in which $N \rightarrow \infty$ while the average degree $N r=z$ is constant. Figure 5 shows that, in this limit, for $z \gg 1$, as in the nonextensive limit with fixed $r$, a third of the eigenvalues are due to Type-I orbits, while virtually none are due to Type-II orbits and the $\lambda=0$ condensate is empty. However, near the giant component transition, $z \sim 1$, the situation changes. Here the fraction of eigenvalues due to Type-I starts to decrease, the $\lambda=0$ condensate starts to fill, and the fraction of eigenvalues due to Type-II orbits reaches a maximum. For $z \ll 1$, the condensate absorbs almost all of the eigenvalues, while the fraction of the eigenvalues that are due to simple orbits vanishes. However, as shown in the inset of Fig. 5 , even for $z \ll 1$, a substantial fraction of the eigenvalues that are not in the condensate are always eigenvalues due to simple orbits. In this limit Type-I and Type-II orbits each produce a quarter of the eigenvalues that are not in the condensate.

In higher dimensional RGGs, similar calculations of the expected number of eigenvalues due to simple orbits are not easy to do exactly. However, what is generally true in higher dimensions is that in the extensive thermodynamic limit in which mean vertex degree is preserved the number of simple orbits and the integer eigenvalues they produce are maximal when the mean degree is near the threshold of bond percolation where the giant component forms. This occurs when the mean degree is of order one. In the limit of low mean degree almost all vertices are isolated and thus almost all eigenvalues are zero and part of the condensate. As mean degree is increased, small clusters of vertices begin to form. Symmetries are plentiful in these small clusters. As the mean degree increases further, the number of components decreases with the giant component dominating the graph, and the number of both Type-I and Type-II orbits decrease. In two-dimensional RGGs, we find numerically that the eigenvalues due to Type-I orbits comprise about $12 \%$ of the spectrum near the giant component transition in connectivity.

The existence of such a large number of orbits has a profound effect on the way that spatial networks behave dynamically. Laplacian spectra describe the normal modes of diffusion on the network, and if the vertices are connected elastically, the normal modes of vibration.
Generally the occurrence of symmetries in a networked system signals that, to linear order, its behavior can be decomposed into a contribution that is confined to a given symmetric structure, and a contribution which affects all vertices in an orbit equally. On the one hand, this impacts controllability of dynamical systems on spatial graphs as the confined modes cannot be excited to linear order by a controller situated elsewhere [28]. On the other hand, the confinement of dynamical modes inside the symmetric structure means that mesoscale structures can exist whose dynamics are largely independent of the embedding network, but which may be able to communicate with the embedding network through nonlinear effects. One can imagine that thereby reusable structures are created that can perform the same function independently of the surrounding networks. Symmetric structures in dynamical networks could therefore have an important effect, for instance, on the evolvability of biological systems. They also have important consequences, for example, in neural networks [22, 23], where an orbit implies that that neurons can be locally excited, and wireless communication networks [13, 29].

The existence of such a large number of orbits in spatial networks can also be used to simplify the analysis of their behavior. By considering a quotient graph [10, 14] the integer eigenvalues can be removed, leaving only the continuous part of the spectrum. The continuous part of the spectrum describes much of the important properties of the network's behavior. For example, the smallest nonzero eigenvalue in graph Laplacian matrices determines the number of vertices or edges that must be cut to sever the network 30]. Also, its eigenvector can be used to partition the network into communities [31]. Notice from Fig. 2 that, in the case shown, pairs of eigenvalues split-off or separate [32, 33] from the bulk continuous distribution at the small end of the spectrum. These separated eigenvalues include the smallest nonzero one. The number of eigenvalues that split off together from the bulk continuous distribution can be deduced by approximating the graph Laplacian with a continuous Laplacian operator corresponding to disordered random media and considering the degeneracy of the modes with smallest eigenvalues [34].

Thus, we have shown analytically that symmetric mesoscale structures are highly abundant for all $r$ in $1 \mathrm{~d}$ RGGs. These motifs lead to integer eigenvalues that comprise a substantial fraction, more than a third, in both intensive and extensive large network limits. Approximate arguments and numerical results indicate that similar behavior occurs in higher dimensional RGGs as well. This behavior differs remarkably from that of non-spatial graphs, which in thermodynamic limits have almost no orbits.

The work of AN and KEB was supported by the NSF through grant DMR-1206839, and by the AFSOR and DARPA through grant FA9550-12-1-0405. 
[1] R. Albert and A.-L. Barabási, Rev. Mod. Phys. 74, 47 (2002).

[2] M. E. J. Newman, SIAM Review 45, pp. 167 (2003).

[3] S. Boccaletti, V. Latora, Y. Moreno, M. Chavez, and D.U. Hwang, Physics Reports 424, 175 (2006).

[4] J. A. Almendral, R. Criado, I. Leyva, J. M. Buldú, and I. Sendiña Nadal, Chaos 21, 016101 (2011).

[5] M. Zanin, P. A. Sousa, and E. Menasalvas, EPL 106, 30001 (2014).

[6] F. Chung, Spectral Graph Theory, vol. 92 of CBMS Regional conference series in mathematics (Conference Board of the Mathematical Sciences, Washington, DC, 1997).

[7] I. J. Farkas, I. Derényi, A.-L. Barabási, and T. Vicsek, Phys. Rev. E 64, 026704 (2001).

[8] L. M. Pecora and T. L. Carroll, Phys. Rev. Lett. 80, 2109 (1998).

[9] P. Mieghem, Graph Spectra for Complex Networks (Cambridge University Press, 2011).

[10] B. D. MacArthur and R. J. Sánchez-García, Phys. Rev. E 80, 026117 (2009).

[11] B. D. MacArthur, R. J. Sánchez-García, and J. W. Anderson, Discrete Applied Mathematics 156, 3525 (2008).

[12] A. Arenas, A. Díaz-Guilera, and C. J. Pérez-Vicente, Physica D: Nonlinear Phenomena 224, 27 (2006).

[13] A. Díaz-Guilera, J. Gómez-Gardeñes, Y. Moreno, and M. Nekovee, International Journal of Bifurcation and Chaos 19, 687 (2009).

[14] H. Aufderheide, L. Rudolf, and T. Gross, New Journal of Physics 14, 105014 (2012).

[15] A.-L. Do, J. Höfener, and T. Gross, New Journal of Physics 14, 115022 (2012).

[16] J. Dall and M. Christensen, Phys. Rev. E 66, 016121 (2002).

[17] M. Penrose, Random geometric graphs, Oxford studies in probability (Oxford University Press, 2003).

[18] M. Barthélemy, Phys. Rep. 499, 1 (2011).

[19] S. Bullock, L. Barnett, and E. A. Di Paolo, Complexity 16, 20 (2010).

[20] M. Haenggi, J. Andrews, F. Baccelli, O. Dousse, and M. Franceschetti, IEEE Journal on Selected Areas in Communications 27, 1029 (2009).

[21] H. Xiao and E. Yeh, Communications Workshops (ICC), 2011 IEEE International Conference on pp. 1-6 (2011).

[22] N. T. Markov, M. Ercsey-Ravasz, D. C. Van Essen, K. Knoblauch, Z. Toroczkai, and H. Kennedy, Science 342 (2013).

[23] M. Ercsey-Ravasz, N. T. Markov, C. Lamy, D. C. V. Essen, K. Knoblauch, Z. Toroczkai, and H. Kennedy, Neuron 80, 184 (2013), ISSN 0896-6273.

[24] D. J. Higham, M. Rašajski, and N. Pržulj, Bioinformatics 24, 1093 (2008).

[25] J. Aguirre, J. M. Buldú, M. Stich, and S. C. Manrubia, PLoS ONE 6, e26324 (2011).

[26] R. Grone and R. Merris, SIAM J. Discrete Math. 7, 221 (1994).

[27] D. M. Cardoso, C. Delorme, and P. Rama, European Journal of Combinatorics 28, 665 (2007).

[28] Y.-Y. Liu, J.-J. Slotine, and A.-L. Barabási, Nature 473, 167 (2011).

[29] N. Fujiwara, J. Kurths, and A. Díaz-Guilera, Phys. Rev. E 83, 025101 (2011).

[30] M. Fiedler, Czechoslovak Mathematical Journal 23, 298 (1973).

[31] D. A. Spielman and S.-H. Teng, Linear Algebra and its Applications 421, 284 (2007).

[32] K. E. Bassler, P. J. Forrester, and N. E. Frankel, Journal of Mathematical Physics 50, 033302 (2009).

[33] K. E. Bassler, P. J. Forrester, and N. E. Frankel, Journal of Mathematical Physics 51, 123305 (2010).

[34] A. Nyberg and K. E. Bassler, unpublished (2014). 